\title{
Transatlantica
}

Revue d'études américaines. American Studies Journal

\section{Journée d'études Historiographie et Militantisme / Historiography and Activism}

Maison de la Recherche de l'Université de Toulouse II-Le Mirail, 11 octobre 2013

Céline Rolland et Lea Stephan

\section{CpenEdition} Journals

Édition électronique

URL : https://journals.openedition.org/transatlantica/6423

DOI : 10.4000 /transatlantica.6423

ISSN : 1765-2766

Éditeur

Association française d'Etudes Américaines (AFEA)

Référence électronique

Céline Rolland et Lea Stephan, « Journée d'études Historiographie et Militantisme / Historiography and Activism », Transatlantica [En ligne], 1 | 2013, mis en ligne le 16 février 2014, consulté le 03 février 2023. URL : http://journals.openedition.org/transatlantica/6423 ; DOI : https://doi.org/10.4000/ transatlantica.6423

Ce document a été généré automatiquement le 3 février 2023.

\section{c) (i) $९$}

Creative Commons - Attribution - Pas d'Utilisation Commerciale - Pas de Modification 4.0 International - CC BY-NC-ND 4.0

https://creativecommons.org/licenses/by-nc-nd/4.0/ 


\title{
Journée d'études Historiographie et Militantisme / Historiography and Activism
}

\author{
Maison de la Recherche de l'Université de Toulouse II-Le Mirail, 11
}

octobre 2013

\section{Céline Rolland et Lea Stephan}

1 Cette journée a été organisée par Nathalie Dessens et Anne Stefani, membres du CAS (Cultures Anglo-Saxonnes), EA 801, Université de Toulouse II-Le Mirail.

\section{Ouverture / Opening}

2 C'est d'emblée de manière assurément subjective qu'Anne Stefani fait débuter la journée, en décrivant les raisons qui l'ont portée à s'intéresser, alors qu'elle n'est pas elle-même militante, à ce que peut signifier l'engagement politique pour l'historien. Elle aborde des problématiques qui reviennent ensuite au fil des communications et sont centrales au thème retenu pour la journée: comment concilier, d'une part, le sacro-saint devoir d'objectivité, voire d'impartialité traditionnellement pensé comme inhérent au travail de l'historien et, d'une autre part, la subjectivité évidente qui intervient dans l'action militante? N'y a-t-il pas un risque de collusion, voire un conflit inévitable, lorsque l'historien affiche ses opinions et s'engage dans la société de son temps pour défendre une cause devenue alors politique?

Dans un second temps, elle invite les participants à s'interroger sur l'influence des stéréotypes socioculturels ou des luttes sociales et politiques sur le travail des chercheurs. Elle cite des ouvrages-clé et pionniers dans l'écriture de l'histoire par des historiens ouvertement engagés et qui proposent leur réflexion sur le sujet, tels The Burden of Southern History de C. Van Woodward, paru en 1960, puis The Southern Mystique (1964) de Howard Zinn dont il sera longuement question plus tard dans la matinée. 
Des historiens tels que Zinn ont mis leur expertise scientifique au service de la cause pour laquelle ils se sont engagés, en l'occurrence le Mouvement pour les Droits Civiques aux États-Unis dans les années 1960. Cela tend à prouver que loin d'être antagonistes, le travail de recherche et l'action militante s'éclairent l'un l'autre.

5 Ainsi, si l'historien peut légitimement choisir d'afficher sa sensibilité politique sans ambiguité, si cela peut même s'avérer intéressant pour mieux écrire et comprendre l'objet étudié, il s'agit, par contre, de s'interroger sur la tendance consistant à ne tenir pour légitime à traiter sérieusement un sujet qu'un chercheur qui serait lui-même socialement immergé dans son sujet d'étude, qui appartiendrait au groupe social dont il défend la cause par ses écrits scientifiques et son militantisme. Pour Anne Stefani, l'excellence vers laquelle tout historien doit tendre s'apprivoise avant tout par un effort de précision, de minutie visant à éviter les malentendus, par un travail de recoupement et de croisement. Il s'agit de donner voix à toutes les parties prenantes.

6 Nathalie Dessens ajoute, au sujet de la période évoquée, que la genèse et même les débuts à part entière du Mouvement pour les Droits Civiques sont bien antérieurs à ce qu'on peut lire ou entendre d'ordinaire, même chez les historiens qui concèdent qu'il faut élargir la période et remontent au début du vingtième siècle. Le Mouvement a démarré en Louisiane pendant la guerre de Sécession.

Elle propose ensuite un résumé de la communication de l'historienne Patricia Sullivan de l'Université de Caroline du Sud qui devait participer à cette journée, mais n'a pas pu être présente. Son intervention portait sur l'inégalité raciale qui perdure aujourd'hui dans la société américaine malgré le symbole fort de l'élection de Barack Obama aux fonctions présidentielles. La conférence initialement prévue, intitulée «What Happened to the Civil Rights Movement? A Reconsideration of C. Vann Woodward's 1967 Essay ", est articulée autour d'un essai de Woodward interprétant le sens et l'évolution du mouvement dont il était contemporain. Patricia Sullivan montre que le Mouvement pour les Droits Civiques est également un mouvement d'ampleur et d'enjeux nationaux et non un phénomène régional limité au Sud du pays.

\section{Conférence / Keynote Lecture : Hélène Le Dantec- Lowry, Université Paris 3, "The Moynihan Report : When Government Meets Activists and Social Scientists »}

Spécialiste de l'histoire socioculturelle des Afro-Américaines, Hélène Le Dantec-Lowry s'intéresse aux répercussions du rapport intitulé The Negro Family: the Case for National Action rédigé par le Démocrate Daniel Patrick Moynihan, qui concerne la forte proportion de foyers afro-américains touchés par le chômage et la pauvreté dans les villes américaines. Commandé par l'Administration Kennedy et publié sous Johnson en 1965 , ce rapport suggère des réformes socio-économiques. Il fut d'abord favorablement reçu avant d'être l'objet de longues controverses, notamment chez les activistes afroaméricains. Il représente la convergence entre la recherche universitaire et le militantisme; de plus, il est le fruit d'une recherche partisane, puisque c'est une commande du Parti démocrate. La vision de la famille idéale - modèle nucléaire de la famille blanche traditionnelle avec un père subvenant seul aux besoins du foyer établi dans la banlieue aisée de la classe moyenne - correspond à l'idéologie de l'époque de la 
Guerre Froide mais pas au modèle culturel afro-américain, ni à la répartition traditionnelle des rôles dans la famille afro-américaine. La pénurie d'emplois masculins est imputée à la montée du secteur tertiaire: les femmes, dans une économie de services, ont moins de difficulté à trouver un emploi, ce qui provoque un déséquilibre que Moynihan pense nuisible à l'estime de soi du père de famille afro-américain car il porte atteinte à sa virilité. Par conséquent, c'est ce déséquilibre qui est, selon Moynihan, néfaste à la stabilité de la cellule familiale. Ce sont donc les femmes qui se retrouvent accusées d' "émasculer» leurs maris et, par là-même, de retarder l'ascension socio-économique de la communauté noire.

9 Mme Le Dantec montre que les activistes afro-américains avaient eux-mêmes un discours semblable à celui de Moynihan que les féministes afro-américaines déploraient mais ne pensaient pas pouvoir combattre sans porter préjudice à la cause noire, jugée primordiale à l'époque.

\title{
Session 1 : L'Héritage du Mouvement pour les Droits Civiques / The Legacy of the Civil Rights Movement
}

\author{
Olivier Maheo, Université Paris 3, « Manning Marable, militant et \\ historien : redéfinir une histoire des Afro-Américains »
}

S'il figure dans la lignée du radicalisme noir depuis Du Bois, Manning Marable est présenté par Olivier Maheo comme le théoricien d'un marxisme noir, inspiré de Gramsci, qui prône un socialisme non sectaire. S'il souhaita dépasser le système bipartite pour proposer une troisième voie, il ne fut pour autant jamais en rupture complète avec le Parti démocrate dont, toute sa vie, il chercha, de l'intérieur, à infléchir la politique vers la gauche. Pour lui, la classe sociale doit primer sur l'appartenance raciale et c'est en ce sens qu'il propose une voie « transformationniste », c'est-à-dire ni "inclusionniste» ou intégrationniste, ni nationaliste. Il est perçu comme un "nationaliste de gauche ", utilisant le discours marxiste afin de mettre en lumière le lien étroit entre racisme et capitalisme. Il redéfinit également les concepts qu'il juge peu opérants pour créer ses propres termes.

11 Ses choix politiques sont, pour Marable, inséparables de sa démarche scientifique : il s'agit de faire en sorte que les Afro-Américains soient à même de prendre le contrôle sur leur propre histoire. Il donne ainsi des cours à la prison de Sing Sing. Sa pensée radicale, reconnue par la recherche universitaire puisqu'il occupe un poste à l'Université de Columbia où il fonde le Département d'Études Afro-américaines et une revue, Souls, prouve que rigueur scientifique et engagement politique se nourrissent l'un l'autre.

\section{Ambre Ivol, Université de Nantes, « Howard Zinn's Politics of History : Reassessing the Current Historiographical significance of A People's History of the United States from 1492 to the Present Day »}

12 Le projet de réécrire une histoire américaine en s'appuyant sur des témoignages et des objets jusqu'alors jugés peu dignes d'intérêt car populaires a produit une œuvre que 
s'est arrachée le grand public américain, à tel point que cette approche est devenue aujourd'hui consensuelle.

Ambre Ivol propose d'éclairer l'enjeu politique de l'œuvre de Zinn, de préciser une typologie des critiques faites à son encontre puis de voir comment il est possible de répondre aujourd'hui à ces critiques. Elle rappelle d'abord la formule de Zinn, «the inevitable taking of sides" : du fait même de la tâche de l'historien, consistant à sélectionner ses sources, ses objets, il doit faire face à la nécessité de prendre parti, de choisir son camp, surtout lorsqu'il entend faire de l' «histoire radicale». Les buts d'Howard Zinn lors de la rédaction étaient d'aiguiser la perception de son lecteur au sujet des victimes de la société américaine, de mettre en lumière l'idéologie véhiculée par les gouvernements, de proposer des possibilités de réforme pour une vie meilleure, d'être honnête au sujet des mouvements sociaux et de dire pourquoi ils peuvent parfois se tromper.

La portée politique de cet ouvrage explique certaines résistances et controverses.

\title{
Session 2: Histoire et Diversité dans l'Amérique Contemporaine / History and Diversity in Today's America :
}

\author{
Hélène Charlery, Université de Toulouse II-Le Mirail, « Bringing the \\ Girls Home : Black Feminist Activism and Visibility in Home Girls \\ (1983-2000)»
}

Hélène Charlery a montré la place de l'anthologie Home Girls: A Black Feminist Anthology publiée en 1983 ainsi que de l'anthologie The Black Woman publiée en 1970 dans la construction du féminisme noir et dans l'affirmation d'un pragmatisme activiste. Ces anthologies avaient été pensées comme un outil politique permettant aux femmes noires de se poser comme agents de l'histoire et d'écrire la culture et l'histoire des femmes noires. Elles représentaient un espace pour la théorisation du féminisme noir ainsi qu'un espace de dialogue avec d'autres groupes militants. Elles se voulaient nonacadémiques et non-élitistes. Toutefois, depuis les années 1990, le sujet a été récupéré par le monde universitaire. Du fait que l'étude des femmes noires se situe à l'intersection des études féministes et des études afro-américaines, elles ont été récupérées par les deux champs d'étude et ne sont plus entre les mains des femmes noires comme cela fut le cas au départ. Bien que le succès des premières éditions ait permis de remarquer qu'il existait une forte demande pour ce type de textes, le constat fut plus mitigé lors des rééditions de Home Girls en 2000 et de The Black Woman en 2005. En effet, la nouvelle préface de Home Girls suggère que l'objectif de donner une visibilité au féminisme noir en dehors du monde universitaire n'a pas été atteint. En revanche, ces rééditions appellent à un renouvellement de ce type d'activisme.

Lors de la discussion, le problème de "l'intersectionalité » a été souligné, ainsi que celui de la réappropriation par ces deux champs d'études. Ainsi, souvent, la particularité de la femme noire, à savoir de réunir les questions de la race, de la classe et du genre, se perd dans la masse de la nouvelle recherche et ce phénomène est renforcé par l'accès limité des femmes noires à la recherche. 


\section{Kenneth Alakson, Union College, United States, «From the Exotic to the American : the Push for Diversity and the Historiography of New Orleans »}

17 Kenneth Aslakson, quant à lui, s'est penché sur la place particulière de la NouvelleOrléans dans l'historiographie. Bien que la Nouvelle-Orléans soit le berceau du Mouvement des droits civiques pendant la guerre de Sécession, elle n'a pas fait l'objet d'études spécifiques jusqu'à la fin du xxe siècle. K. Aslakson lie cela à la nature exceptionnelle de la Nouvelle-Orléans marquée par la présence de son importante population de Créoles de couleur. Le regard sur la Nouvelle-Orléans n'a changé que dans le sillage du Mouvement pour les droits civiques et du remplacement dans l'histoire et la société américaines de l'idéal du melting pot par celui d'une Amérique pluriculturelle. Le nouveau regard porté sur la population noire, qui n'est plus considérée comme monolithique mais qui est envisagée sous toutes ses facettes et dans toute sa diversité, ont changé le regard sur la Nouvelle-Orléans. De plus en plus, la Nouvelle-Orléans est vue comme une partie du Sud parmi d'autres, et non comme une exception, comme un élément difficile à classer.

Lors de la discussion, la question de la définition de qui est noir ou non a resurgi en lien avec la Nouvelle-Orléans, en particulier en ce qui concerne les nouveaux immigrants africains aux États-Unis. De plus, ces questions ont réorienté le débat vers le processus de création d'identité, en particulier la part subjective et la part imposée par autrui et la société.

\section{Sonia Rose, Université de Toulouse II-Le Mirail, « Latin American Scholars in the United States: Historicising (Post)coloniality the South American Way »}

Sonia Rose a étudié les changements dans la discussion historiographique concernant le concept de "période coloniale» appliqué à l'Amérique Latine. Son groupe de référence était composé d'hommes et de femmes nés en Amérique Latine, mais qui occupaient des positions dans des universités de l'Ivy League aux États-Unis entre la fin des années 1980 et 2013. Elle a soulevé la question de la représentation et de la légitimité de l'expression de ces enseignants-chercheurs. De plus, elle a insisté sur le fait que ces universitaires communiquaient très peu avec leurs homologues en Amérique Latine, et s'adressaient directement aux populations indigènes, ce qui se traduisait notamment par une collaboration étroite avec la Andean Indigenous University à Quechua. Cette collaboration entre des universités élitistes et une université à l'accès très réduit, de par sa langue hautement spécialisée et peu parlée, renforçait non seulement le caractère problématique des représentations, mais posait aussi la question du public à laquelle s'adressait cette recherche.

La discussion s'est concentrée, une fois de plus, sur la relation entre le monde universitaire et l'activisme, et sur la perte éventuelle de l'autorité de la parole lorsqu'un universitaire devenait activiste. 


\section{Conclusion}

21 Lors de la première session, c'est la difficulté de concilier une position ouvertement militante et un travail objectif de chercheur qui a primé. La seconde session a permis la mise en lumière d'une autre question de statut : comment respecter, pour l'historien, les appartenances diverses de son sujet d'étude lorsque ce sujet humain se situe à la croisée de plusieurs catégories et qu'il apparaît donc difficilement classable.

INDEX

Thèmes : Actualité de la recherche

\section{AUTEURS}

CÉLINE ROLLAND

Université de Toulouse II-Le Mirail

\section{LEA STEPHAN}

Université de Toulouse II-Le Mirail 